Methods A total of 104,333 eligible males enrolled in every 2-year health checkup were involved in the Kailuan male cohort study (2006-2015). Cox proportional hazards regression models were used to estimate the association between components of MetS and the CRC risk

Results During a median follow-up of 8.9 years, 394 CRC cases were verified over a total of $824,211.96$ person-years. Compared with males without MetS components, the HRs (95\% CI) of developing CRC for males with 1,2 and $\geq 3$ MetS components were 1.53 (1.01-2.32), 1.42 (0.94-2.14) and $1.70(1.12-2.56)$, respectively, which showed a statistically trend $(\mathrm{P}$ trend $=0.04)$ for increasing risk with an increasing number of MetS components. In addition, among the 5 single MetS components, only the waist circumference $\geq 90 \mathrm{~cm}$ showed significant risk for CRC (HR: 1.32, 95\% CI: 1.071.64). Further combination of higher waist circumference and elevated fasting blood glucose $(\geq 5.6 \mathrm{mmol} / \mathrm{L}$ or drug treatment of elevated glucose) with normal levels of the other three components showed an increased risk for CRC by $126 \%$ (95\% CI: 1.10-4.64). However, the other combinations showed non statistical significant associations.

Conclusions Our study suggests CRC risk is correlated with the number of abnormal MetS components in males. Among MetS components, higher waist circumference, particularly concurrent with elevated fasting blood glucose may be more strongly related to CRC risk than other MetS components.
IDDF2019-ABS-0182 A CLOSTRIDIA-RICH ENTEROTYPE CONTRIBUTES TO INCREASED EXCRETION OF BILE ACIDS IN DIARRHEAPREDOMINANT IRRITABLE BOWEL SYNDROME

Ling Zhao*. School of Chinese Medicine, Hong Kong Baptist University, Hong Kong

\subsection{6/gutjnl-2019-IDDFabstracts.34}

Background An excess of fecal bile acids (BAs) is thought to be one of the peripheral mechanisms for diarrhea-predominant irritable bowel syndrome (IBS-D). However, the factors causing excessive BA excretion remains unclear. Given the importance of gut microbiota in BA metabolism, we hypothesized that gut dysbiosis might contribute to excessive BA excretion in IBS-D.

Methods Metabolomic and metagenomic analyses were performed of specimens from 290 IBS-D patients and 89 healthy volunteers. By transplanting human microbiota and manipulating specific bacterial species in mice, the effects of microbiota on host BA metabolism were assessed at metabolic, genetic and protein levels. Effects of individual and mixed BAs on enterohepatic feedback pathways were also tested in vitro and in vivo.

Results Total fecal BAs were excessively excreted in 71 of 290 IBS-D patients (grouped as $\mathrm{BA}^{+} \mathrm{IBS}-\mathrm{D}$ ) who also have excessive $7 \alpha$-hydroxy-4-cholesten-3-one (C4) but deficient fibroblast
A

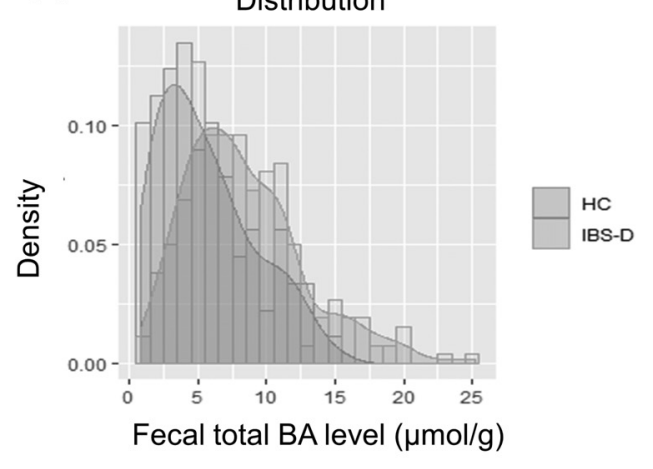

D

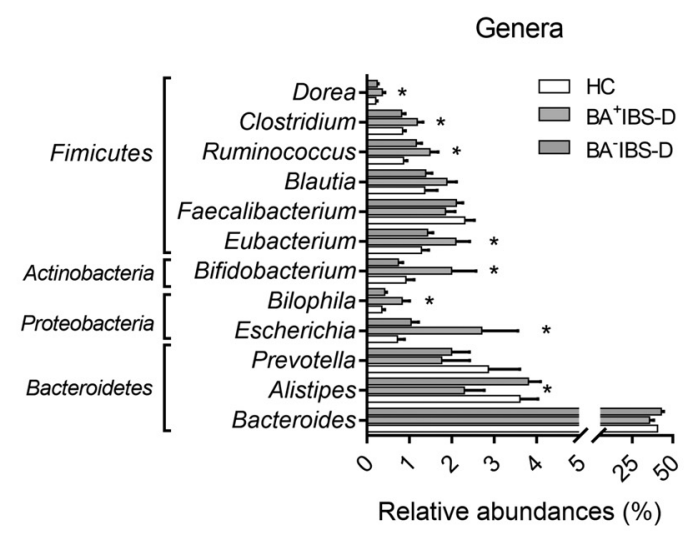

B

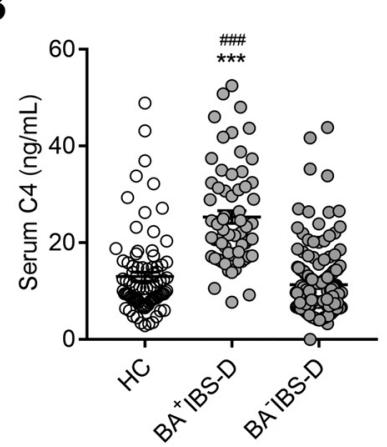

E

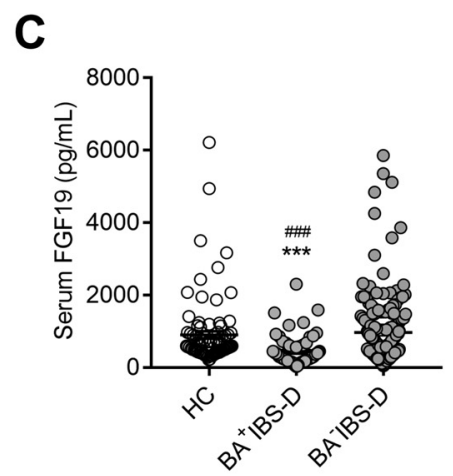

Correlation coefficient

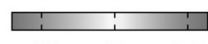

$\begin{array}{lll}-0.2 & 0 & 0.2\end{array}$

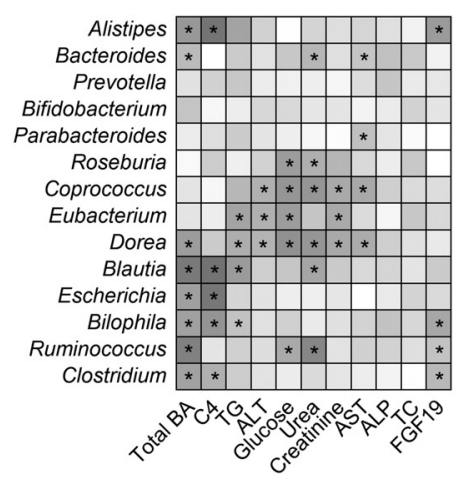

Abstract IDDF2019-ABS-0182 Figure 1 
growth factor 19 (FGF19) in sera (Figure 1A-C. The association of clostridia rich microbiota with BA synthesis and excretion in IBS $d$ patients a the histogram of the distribution of total fecal BA level in recruited subjects $\underline{b} \mathbf{c}$ concentrations of C4). Their fecal metagenomes showed increased abundances of Clostridia and BA-transforming genomes ( $h d h A$ and bais). The increase of Clostridia (e.g. Clostridium) was positively associated with the levels of fecal BAs and serum $\mathrm{C} 4$, while being negatively correlated with serum FGF19 (Figure 1D, E). However, there is no big difference in BA profile and BA-transforming microbiome found in other IBS-D patients with normal BA excretion (grouped as BA-IBS-D) relative to controls. Further, transplantation of Clostridia-rich microbiota from IBS-D donors and introduction of a BA-transforming Clostridium species both enhanced levels of serum C4 and hepatic conjugated BAs in mice and reduced ileal FGF19 expression. Inhibition of Clostridium species by vancomycin yielded opposite findings. Moreover, Clostridia-derived BAs, like conjugated and free ursodeoxycholic acid, were also found to be significantly suppressed intestinal FGF19 expression in vitro and in vivo.

Conclusions The Clostridia-rich microbiota contributes to excessive BA excretion in IBS-D patients. This study provided the basis for more precise clinical diagnosis and management for IBS-D.

\section{IDDF2019-ABS-0184 ROLE OF ADHERENT-INVASIVE E. COLI IN INFLAMMATORY BOWEL DISEASE - EPIDEMIOLOGY, GENETICS AND THERAPEUTICS}

'Zhilu Xu*, 'Keli Yang, 'Jingwan Zhang, ${ }^{1}$ Tao Zuo, ${ }^{2}$ Caroline Chevarin, ${ }^{1}$ Lok Cheung Chu, ${ }^{1}$ Francis Chan, ${ }^{1}$ Joseph Sung, ${ }^{1} J u n$ Yu, ${ }^{2}$ Nicolas Barnich, ${ }^{1}$ Sunny Hei Wong, ${ }^{1}$ Siew Chien Ng. ${ }^{1}$ Department of Medicine and Therapeutics, Institute of Digestive Diseases, LKS Institute of Health Sciences, State Key Laboratory of Digestive Disease, The Chinese University of Hong Kong, Hong Kong; ${ }^{2}$ Universite Clermont Auvergne, Inserm U1071, USC-INRA 2018, M2iSH, F-63000, France

\subsection{6/gutjnl-2019-IDDFabstracts.35}

Background Adherent-invasive Escherichia Coli (AIEC) colonizes mucosa of patients with Crohn's disease (CD). Fecal microbiota transplantation (FMT) has been reported to be effective in treating $\mathrm{CD}$ but the response rates are variable. We investigated the prevalence of AIEC in Chinese population and explored the impact of AIEC on FMT efficacy.

Methods AIEC strains were isolated from ileal tissues of Chinese Crohn's disease patients $(n=64)$ and healthy subjects $(n=53)$ by selection culture and protection assay in Intestine-407 cell lines and their genomes analysed. Effect of AIEC on FMT was assessed in C57BL/6 wild type DSScolitis model. Level of AIEC colonization was assessed by selective culture and Fluorescence in situ hybridization. Human ileal tissue and mice feces were collected for $16 \mathrm{~S}$ rRNA sequencing.

Results Prevalence of AIEC was significantly higher in mucosa of CD subjects than healthy controls $(p<0.05)$. Significant loss of Megamonas and Veillonella was observed in AIEC-positive ileal tissues, compared with AIEC-negative and healthy tissues. The majority of AIEC isolates belonged to B2 phylogroup. 43.9\% of AIEC isolates were multi-drug-resistant. The Chinese AIEC isolates possessed a significantly higher prevalence of Quinolone-, Macrolide-, Sulphonamide-, Trimethoprim-, and Tetracycline- resistant genes, compared with previously reported strains. These antibiotics resistant strains possessed significantly higher invasion properties than did non-resistant strains. In mice, FMT was not able to eradicate AIEC from the colon. After FMT, K12 (a noninvasive E. coli strain) - colonised mice, but not AIEC-infected mice, showed ameliorated colitis with elongated colon, improved colonic histology and decreased fecal Lcn-2 levels. FMT increased microbial diversity in $\mathrm{K} 12$-colonised mice but not in AIEC-infected mice. The proportion of donor-derived microbes in K12colonised mice was significantly higher than in AIECinfected mice, where a lack of engraftment of Fecalibacterium prausnitzii, Akkermansia muciniphila and Allobaculum was seen in AIEC-infected mice.

Conclusions AIEC is a risk factor for CD in the Chinese population. The presence of AIEC was sufficient to compromise the efficacy of FMT by hindering the engraftment of beneficial bacteria, leading to incomplete recovery of inflammation.

\section{IDDF2019-ABS-0188 GUT MICROBIOTA MODULATES THE CHEMOPREVENTIVE EFFICACY OF ASPIRIN ON COLORECTAL CANCER THROUGH IMPACT ON ASPIRIN BIOAVAILABILITY}

${ }^{1}$ Risheng Zhao*, ${ }^{1}$ Olabisi Coker, ${ }^{2}$ Jianlin Wu, 'Liuyang Zhao, ${ }^{1}$ Geicho Nakatsu, ${ }^{2}$ Xiqing Bian, ${ }^{1}$ Joseph Sung, ${ }^{1}$ Francis Chan, ${ }^{3}$ Emad El-Omar. ${ }^{1}$ The Chinese University of Hong Kong, Hong Kong; ${ }^{2}$ Macau University of Science and Technology, Macau; ${ }^{3}$ University of New South Wales, Australia

\subsection{6/gutjnl-2019-IDDFabstracts.36}

Background The gut microbiota, a pivotal regulator in colorectal cancer (CRC) development, is profoundly involved in drug metabolism. This study aims to decipher the role of the gut microbiota in aspirin-mediated chemoprevention of CRC in mice. Methods Two models, azoxymethane (AOM, $10 \mathrm{mg} / \mathrm{kg}$ ) and dextran sulfate sodium (DSS, 2.0\%) induced CRC and APC$\mathrm{min} /+$ induced CRC, were used in this study. Mice were orally administered aspirin $(400 \mathrm{mg} / \mathrm{L})$ with or without periodical antibiotics cocktail $(0.1 \mathrm{~g} / \mathrm{L}$ of vancomycin, $0.2 \mathrm{~g} / \mathrm{L}$ of ampicillin, metronidazole and neomycin). AOM/DSS-treated germ-free mice and conventionally reared germ-free mice (conventionalized mice) were used for validation. Aspirin concentration was estimated by ultra-high performance liquid chromatography (UHPLC). Aspirin-degrading microbes were identified by UHPLC and16S rRNA gene sequencing.

Results The tumor number and load were significantly reduced after aspirin treatment in antibiotics-treated mice in both AOM/DSS-treated and APC ${ }^{\text {min/+ }}$ models, but not significantly changed in non-antibiotics-treated mice. Consistently, aspirintreated germ-free mice exhibited significantly less tumor number and load compared to those not receiving aspirin, which was not seen in conventionalized mice, suggesting that gut microbiota impairs the chemopreventive efficacy of aspirin on CRC. UHPLC analysis revealed that plasma levels of aspirin were significantly higher in microbiota-depleted mice compared to microbiota-intact mice, indicating that gut microbiota limits aspirin bioavailability. Co-incubation of aspirin-containing medium and bacteria showed that aspirin levels in medium were significantly reduced when incubated with the fecal commensal bacteria from microbiota-intact mice compared to those from microbiota-depleted mice. Screening 1,093 bacterial colonies isolated from the feces of microbiota-intact mice 